\title{
Trifunctional antibodies unleash NK cells
}

Combining
the three
functionalities
in one
molecule was
also more
efficient
than mixing
bispecific
antibodies ...

The concept of engineering multifunctional antibodies that tether killer cells to cancer cells is not new, and the first bispecific antibody, blinatumomab, binding to both the $\mathrm{T}$ cell receptor and the $\mathrm{B}$ cell antigen CD19, received FDA approval in 2017. However, therapeutic approaches that induce powerful $\mathrm{T}$ cell responses can also lead to life-threatening cytokine-release syndrome in patients. Now, Eric Vivier and colleagues report the development of trispecific antibodies that engage two activating receptors on natural killer (NK) cells as well as a tumour-specific antigen and show that this ' $\mathrm{NK}$ cell engager' (NKCE) has potent activity in in vivo models.

NK cells express a number of activating receptors, including $\mathrm{CD} 16$ (also known as Fc $\gamma$ RIIA), NKG2D, SLAM family members and the natural cytotoxicity receptors NKp30, NKp44 and NKp46. Of these, NKp46 was identified as a particularly promising target because it is frequently expressed by tumour infiltrating NK cells and sustained expression of NKp46, associated with the downregulation of other activating receptors, has been reported for tumour infiltrating NK cells in several types of human cancer.

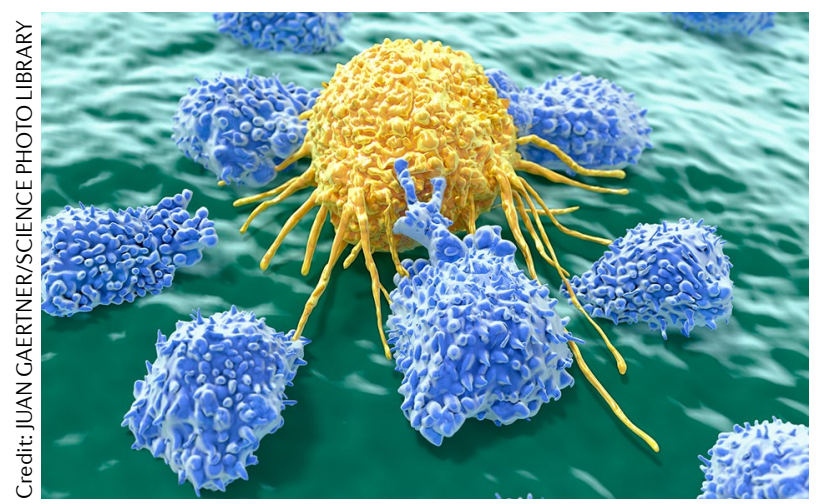

The authors first generated a number of anti-NKp46 antibodies, the most promising of these were humanized and characterized in detail. Next, a small selection of these were turned into bispecific antibodies by associating their Fab fragments (containing the antigen-specific variable region) with single-chain variable fragments (scFvs) directed against the tumour antigen of interest, such as CD19. These bispecific antibodies lacked the nonvariant fragment crystallizable (Fc) domain that binds to CD16 on NK cells ('Fc silent'). The NK cell activating and subsequent tumour killing activity of these bispecific antibodies was compared to an anti-CD19 IgG1 antibody that induces antibody-dependent cell-mediated cytotoxicity (ADCC) by activating NK cells via CD16. In vitro experiments with purified resting NK cells and B cell lymphoma cells showed that all the bispecific antibodies were more effective at inducing tumour cell killing than the anti-CD19 antibody, even though the expression of CD16 on NK cells was higher than the expression of NKp46. This indicated that engaging NKp46 is an efficient way of activating NK cell-mediated ADCC.

Next, a bispecific Fc-silent antibody targeted at the $B$ cell antigen CD20 and NKp46 was tested in a mouse model of lymphoma. It was shown to promote NK cell infiltration into the tumour and NK cell mediated tumour clearance. Finally, the bispecific format was turned into a trispecific format by incorporating an optimized Fc portion that efficiently engages CD16 on NK cells. These NKCEs were engineered to target CD19, CD20 or the lung carcinoma antigen
EGFR and compared with NKCEs with silent or non-optimized Fc domains. In vitro experiments showed that Fc optimized trifunctional NKCEs are particularly efficient at NK cell activation and tumour cell lysis, regardless of the tumour antigen targeted. Combining the three functionalities in one molecule was also more efficient than mixing bispecific antibodies targeting the tumour antigen and either NKp46 or CD16 separately.

Remarkably, when incubated with purified NK cells and the respective tumour cells, the trifunctional NKCEs were more efficient at inducing tumour cell killing than the anti-CD20 targeted antibodies rituximab and obinutuzumab or the anti-EGFR antibody cetuximab, which are widely used in the clinic. Importantly, there was no evidence of off-target effects on antigen-negative cells or of NK cell versus NK cell fratricidal killing.

Finally, the CD20-targeted Fc-optimized trispecific NKCEs (CD20-NKCEs), CD20-targeted Fc-silent bispecific NKCEs and obinutuzumab were compared in two different models of B cell lymphoma. The CD20-NKCEs proved to be most potent and significantly more efficient at inducing tumour cell killing than obinutuzumab over a range of doses. The potential for toxicities was assessed in a cytokine release assay using human peripheral blood mononuclear cells, in which CD20-NKCEs induced barely detectable cytokine release, with a similar cytokine-release profile to obinutuzumab.

Together, these results suggest that trispecific NKCEs efficiently harness the antitumour efficacy of NK cells and support the clinical development of this new modality for cancer immunotherapy.

Alexandra Flemming

ORIGINAL ARTICLE Gauthier, L. et al. Multifunctional natural killer cell engagers targeting NKp46 trigger protective tumor immunity. Cell https://doi.org/10.1016/ j.cell.2019.04.041 (2019) 